\title{
Analytical Approximations and Simulation Tools for Water Cooling of Hot Rolled Steel Strip
}

\author{
Aarne Pohjonen ${ }^{1} \quad$ Vesa Kyllönen $^{2} \quad$ Joni Paananen $^{1}$ \\ ${ }^{1}$ Materials and Production Technology, University of Oulu, Finland, \\ Aarne.Pohjonen@oulu.fi, Joni.Paananendoulu.fi \\ ${ }^{2}$ Technical Research Centre of Finland, VTT, Finland, Vesa.Kyllonen@vtt. fi
}

\begin{abstract}
Analytical approximations that can be used together with the numerical codes to obtain estimates on the temperature distribution inside of the cooled steel strip/plate are discussed. While numerical simulations can give accurate answer after the time required for calculations, the analytical approximations show how thickness and cooling rate affect the temperature distribution. We also present development of graphical user interface interaction with numerical codes for the use in designing and tuning of water cooling schedule for hot rolling strip and plate mill. Interaction of the numerical codes with user friendly frontends have been developed for the following tools: a heat conduction simulation tool for hot strip mill, a tool for calculating phase transformations for user defined cooling paths and a tool for calculating the required cooling water to cool a steel strip to a desired temperature. The functionality and interaction of the tools with the numerical codes is described.
\end{abstract}

Keywords: analytical approximation, simulation, graphical user interfaces, steel, steel processing, heat Treatment, cooling

\section{Introduction}

Simplified analytical approximations provide an useful way for quickly estimating physical processes and interpreting results of detailed simulations or complicated theory. The complexity of conduction of heat inside of a steel strip, taking in to account the heat released in phase transformations, the effect of transformations and temperature on the heat conductivity, heat capacity and density make the exact solution viable only through numerical calculations. However, simplified analytical results can be obtained when suitable approximations are made. With the aid of equations presented in this article, it is easy to understand how the cooling rate on the surfaces of the steel strip/plate and the thickness of the strip/plate affect the temperature distribution inside of the strip/plate.
Graphical user interface (GUI) provides other users a possibility of using advanced numerical codes without the need to spend time learning the specific, often cumbersome textual syntax, of a given code. GUI makes it easier to share the knowledge of the scientists or engineers to the user, who does not need to know the exact inner workings of the complex numerical code or analytical theory in order to benefit from the calculations in his/her work. The real industrial data can be automatically read in to the code and the user can easily experiment with the data by changing parameters from the interface. In this way the user can quickly compare numerical simulation data and experimental data to his/her experience and hypotheses. Such interaction shortens the development time from idea to experimentation as well as the number of experiments required, as many things can be experimented easily with numerical codes. Also the development of the code and theory becomes easier when the results can be compared easily to actual measured values obtained either from an industrial process or from experiments.

As part of the current development of simulation tools for the microstructural development of hot rolled steels, we have constructed graphical interfaces for three different tools. A numerical heat conduction tool (Pyykkönen et al. 2010; Pyykkönen et al. 2012a; Pyykkönen et al. 2012b; Pyykkönen et al. 2013) has been connected to a web based graphical user interface that receives real industrial data and allows the user to change the input from the data. A phase transformation model, which is currently under further development, is connected to GUI that allows user to define a cooling path by clicking with a mouse on a time-temperature diagram and calculate the phases transformed when the steel is cooled along this path. Also, GUI is created for a tool that allows user to define desired temperature path for cooling of steel and calculates the required water usage to cool the steel to this temperature. 


\section{Analytical approximations for the temperature distribution during cooling}

To find an estimate for the temperature distribution for a given cooling rate, we seek a time-asymptotic solution $T_{A}$ to the heat equation with linear cooling rate specified at the top $(x=L)$ and the bottom $(x=-L)$ of a strip/plate, and discuss the time required for the material to converge to this temperature distribution, and the transient temperature distribution from initial distribution to the time-asymptotic distribution.

The time dependent temperature distribution within material can be solved from the heat equation

$$
\rho c \frac{\partial}{\partial t} T(\vec{x}, t)-\nabla \cdot(\kappa \nabla T(\vec{x}, t))=\sigma,
$$

where $\rho$ is density, $c$ is the specific heat capacity, $\kappa$ is the heat conductivity, $\sigma(x, t)$ is amount of heat generated per time unit and $T(\vec{x}, t)$ is the temperature distribution. The heat conduction within a steel strip can be approximated as 1-dimensional heat conduction, except near the edges of the strip, with heat transfer or temperature described at the top and bottom boundaries of the strip.

In order to obtain simplified analytical expression for the temperature distribution, we additionally assume that the strip is homogenous, the heat conduction, density and heat capacity are constants, and the heat release from the transformations can be neglected. This condition applies for the cooling before and after the transformations, and also when the transformation rate or heat released from given transformation is low. Heat release could be in principle estimated by adding a term corresponding to the heat release to the solution described here, but this is not considered in this work. With these approximations, the 1-dimensional heat equation is described by

$$
\frac{\partial}{\partial t} T(x, t)-k \frac{\partial^{2}}{\partial x^{2}} T(x, t)=0,
$$

where $k=\kappa /(\rho c)$.

Assume that $T_{0}(x)$ is the initial temperature distribution inside of the strip/plate. The full solution $T(x, t)$ to (2) can be written as a sum of the asymptotic solution $T_{A}(x, t)$ and a transition function $T_{T}(x, t)$ that describes the transition from initial temperature distribution to the time-asymptotic solution, as described by

$$
T(x, t)=T_{A}(x, t)+T_{T}(x, t) .
$$

We consider the case that the constant cooling rate is specified on the top and at the bottom of the strip/plate with boundary conditions described by (for generality),

$$
\begin{gathered}
T(-L, t)=c_{0}+c_{1} t, \\
T(L, t)=d_{0}+d_{1} t,
\end{gathered}
$$

Where $c_{l}$ and $d_{l}$ are negative for cooling and positive for heating. It can be verified by direct substitution that the time-asymptotic Taylor series solution, $T_{A}$ to (2) with boundary conditions given by (4) and (5) is described by

$$
\begin{aligned}
& T_{A}(x, t)=\frac{\left(c_{1}+d_{1}\right)}{2} t+\frac{c_{0}+d_{0}}{2}-\frac{\left(c_{1}+d_{1}\right) L^{2}}{4 k}+\left[\frac{d_{1}-c_{1}}{2 L}+\frac{d_{0}-c_{0}}{2 L}+\frac{\left(c_{1}-d_{1}\right) L}{12 k}\right] x \\
& +\frac{c_{1}+d_{1}}{4 k} x^{2}+\frac{d_{1}-c_{1}}{12 k L} x^{3} .
\end{aligned}
$$

This solution corresponds to case where the material has been cooled long enough, with constant rates on top and bottom of the strip/plate, in order for the distribution to relax to it (there is only linear time dependence in the solution and boundary conditions).

The solution can be further simplified if top and bottom cooling rates are the same and both ends are at the same temperature when $t=0$, i.e. $c_{0}=d_{0}$ and $c_{1}=d_{1}$, which yields

$$
T_{A}(x, t)=c_{0}+c_{1} t-\frac{c_{1} L^{2}}{2 k}+\frac{c_{1}}{2 k} x^{2} .
$$

The equation (7) provides useful approximate estimate for the time asymptotic temperature distribution inside of the strip/plate, which is being cooled at rate $c_{1}$ at both ends.

We also wish to estimate the temperature distribution during the transition from the initial temperature distribution to the time-asymptotic solution (7), and especially the time required for the temperature distribution converge to the time-asymptotic solution. We assume that $T_{0}(x)$ is the initial temperature distribution inside of the strip/plate, with top and bottom of the strip/plate at temperature $c_{0}, T_{0}(-L)=c_{0}$ $=T_{0}(L)$. We denote the $T(x, t)$ as the full time-dependent solution to the differential equation (2). In (Carslaw and Jaeger 1989), time-dependent solutions to this type of problems are given. The solution can be written in the following form

$$
T(x, t)-c_{0}=u(x, t)+w(x, t),
$$

where the functions $u(x, t)$ and $w(x, t)$ are such that

$$
\begin{gathered}
u(x, t)=0, \text { when } x=-L \text { and } x=L, \\
u(x, t)=T_{0}(x)-c_{0}, \text { when } t=0
\end{gathered}
$$

and

$$
w(x, t)=c_{1} t \text {, when } x=-L \text { and } x=L,
$$




$$
w(x, t)=0 \text { when } t=0 .
$$

The functions $u(x, t)$ and $w(x, t)$ are then given by (9) and (10) (Carslaw and Jaeger 1989)

$$
u(x, t)=\frac{1}{L} \sum_{n=1}^{n=\infty} B_{n} \sin \left(\frac{n \pi(x+L)}{2 L}\right) \exp \left(-\frac{k n^{2} \pi^{2}}{4 L^{2}} t\right),
$$

where

$$
B_{n}=\int_{-L}^{L}\left(T_{0}(x)-c_{0}\right) \sin \left(\frac{n \pi(x+L)}{2 L}\right) d x
$$

and

$$
\begin{aligned}
& w(x, t)=c_{1} t+\frac{c_{1}\left(x^{2}-L^{2}\right)}{2 k} \\
& +\frac{16 c_{1} L^{2}}{k \pi^{3}} \sum_{n=0}^{n=\infty} \frac{(-1)^{n}}{(2 n+1)^{3}} \cos \left(\frac{(2 n+1) \pi x}{2 L}\right) \exp \left(-\frac{k(2 n+1)^{2} \pi^{2}}{4 L^{2}} t\right) .
\end{aligned}
$$

The time dependence of (9) and (10) shows that the terms in the solution converge towards the timeasymptotic solution (7), proportionally to $\exp \left(-t / \tau_{n}\right)$, where the time constant $\tau_{n}$ is given by

$$
\tau_{n}=\frac{4 L^{2}}{k n^{2} \pi^{2}},
$$

where $n=1$ corresponds to the slowest time convergence, and can be used to evaluate the time required for the initial temperature distribution to converge towards the time asymptotic temperature distribution given by (7).

\section{Tools}

\subsection{Heat conduction tool for the hot rolled strips}

In order to be able to estimate the time dependent temperature distribution inside of a steel strip, a numerical heat conduction model has been earlier developed (Pyykkönen et al. 2010; Pyykkönen et al. 2012a; Pyykkönen et al. 2012b; Pyykkönen et al. 2013).

Temperature variations within a steel strip cause the phase transformations to start and proceed at different rates. This can cause inhomogenieties in the final microstructure, which affect the flatness and mechanical properties especially for thicker strips or plates.

A web based graphical user interface was developed to interact with the numerical code for the purpose that the development and operation engineers would be able to benefit from the simulation results easily. The graphical user interface is connected to a server side software (see Figure 1) that has two main functions: reading factory data for desired product and start numerical heat conduction simulation corresponding to the industrial strip parameters (strip thickness, water usage at different points along the cooling line etc.).
Using the GUI, the user can modify the actual values that had been used in the real cooling of the strip. The parameters that are used in the detailed cooling model are: the cooling start temperature and the strip speed, cooling water usage at different parts of the cooling line. The model outputs the temperature in the middle of the strip, at quarter thickness and on the top and bottom surfaces as well as at user-defined depth. The output of the simulation is displayed on the

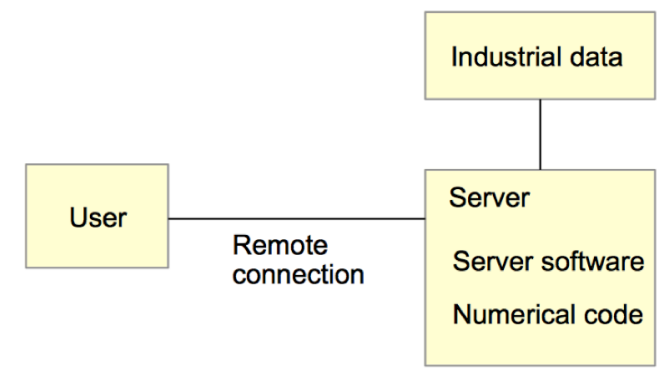

Figure 1. Web browser is used to access the GUI provided by the server software. The server software reads in industrial data, which is used as input for the numerical calculations. The user can modify the input for experimenting how different parameters affect the strip temperatures.

\section{GUI as shown in Figure 2.}

The parameters are passed from the GUI to the numerical heat conduction and phase transformation model as arguments after the executable name in Linux operating system. Following this approach, no swap files need to be generated when starting the computations and further interaction is relatively easy to implement in the codes by adding more arguments to the passing and receiving software. The numerical software produces output files that are then read in and presented by the GUI to the user.

While developing the GUI several project meetings were held where the production and development engineers could suggest modifications to the GUI. This ensured that the functionality provided by the GUI would have the relevant features for the users.

\subsection{Phase transformation calculation along user defined cooling path}

A phase transformation model, which is currently under active development, is coupled to a graphical user interface, which allows user to define time dependent cooling path for the steel. Such model is useful in finding suitable cooling path that leads to desired final phase composition and mechanical properties of the product. The phase transformation model is fitted for given steel composition and thermomechanical treatment in order to obtain as good estimate for the actual transformation behavior of the steel as possible. With numerical experiments using different cooling paths, the time required and cost caused by real experiments can be minimized. The GUI can enable the development engineers to apply even 


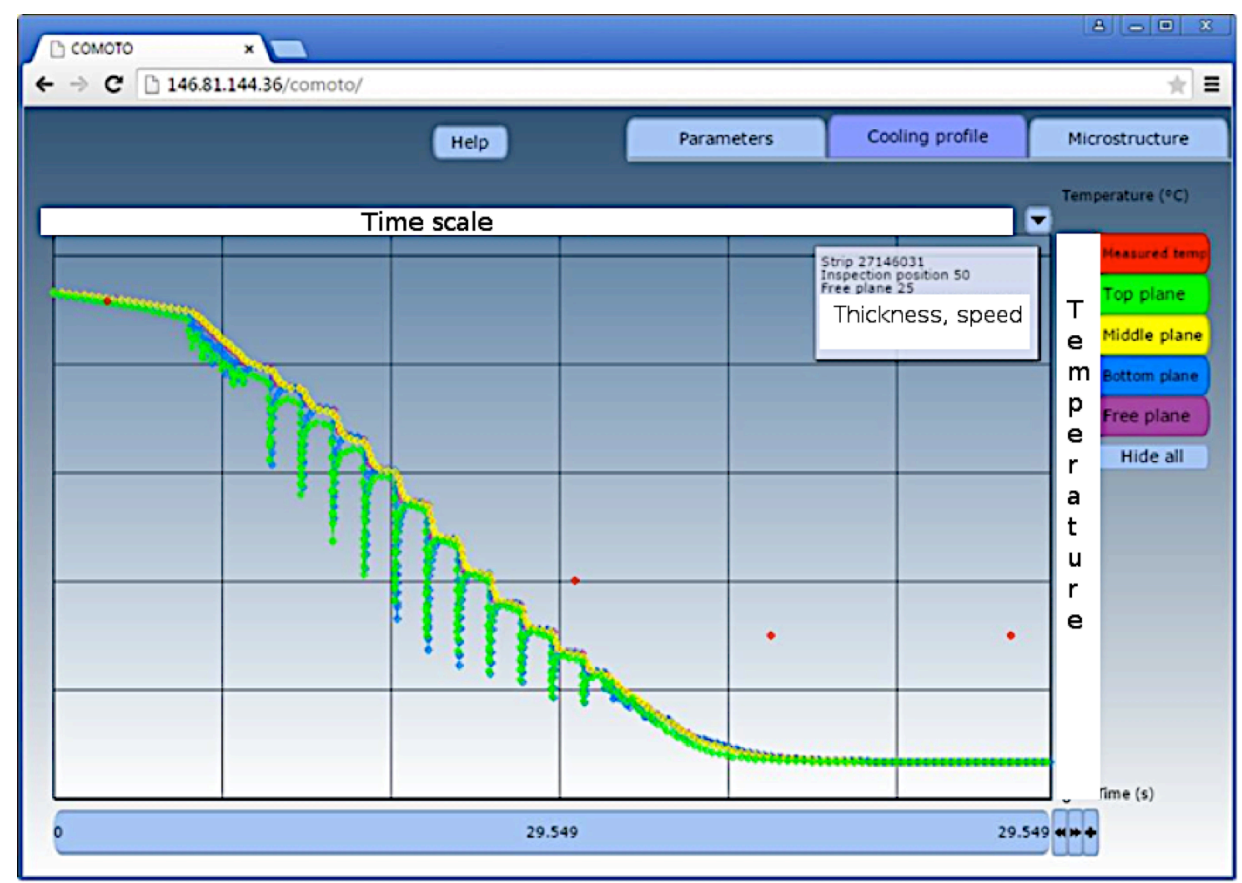

Figure 2. Output from the heat conduction simulation program is visualized with the GUI. The view provides information on the temperature distribution as a function of time.

complicated theoretical transformation model easily. This also benefits the development of the model, as experimental data can be easily compared against the model results.

The graphical user interface is shown in Figure 3. It consists of a diagram with time on the $\mathrm{x}$-axis and temperature on the $y$-axis. Isothermal transformation start curves of ferrite and bainite are plotted in the diagram to show quickly the transformation start during isothermal holding at different temperatures. The user can define the cooling path by selecting points with a mouse on the diagram. While constructing the cooling path, the user can at any point choose calculate how large fraction of austenite has transformed in to ferrite, bainite, pearlite or martensite.

This GUI was developed with Python programming language with using the modules tkinter and subprocess (python documentation). The tkinter module provides simple syntax for several useful commands, such as drop menu, dialog for saving and loading results, buttons, and canvas for drawing. Simple graphical user interface can be generated quickly, and the modularity of the software provides possibility to further development and combining it with larger projects. Because the syntax of Python language is minimalistic, it provides possibility for rapid implementation.

The phase transformation model on the other hand is developed with Fortran 90 programming language, which enables fast numerical calculations and the use of several optimized numerical libraries. In this case the data is passed from the GUI to the transformation model as a single array in a swap file. The chemical composition is read from an input file. The transformation model outputs the results to a file that is in turn read by the Python code. While the combination of the ease of programming with the Python language and the speed of compiled Fortran code utilizes the main advantages of the both languages and is well suited for small scale projects, such as the one presented here, it may be more difficult to implement in more complicated projects that require more interaction between the GUI and the numerical backend software. An article where the details of the renewed phase transformation model are described is under preparation and is submitted for publication in 2017 by the first author of this article.

\subsection{Calculation of water usage required to cool the mid-depth of a steel strip to a desired temperature}

Although the water usage, which is required to cool a steel strip to desired temperatures along the cooling line, could in principle be found by experimenting with different amounts of water and applying the computational method described in section 3.1, this would be cumbersome and slow, since the detailed heat conduction calculation takes 1-5 minutes to complete, depending on the strip thickness. In order to obtain a quick estimate we have developed an approximate method, which calculates how much cooling water is required for cooling the strip at desired temperatures along the cooling line. The model was parameterized using the more detailed model described in section 3.1. The details of this approximate model are presented elsewhere (Paananen 2015, Pohjonen 2016) but here we describe the operation and implementation of the GUI. 


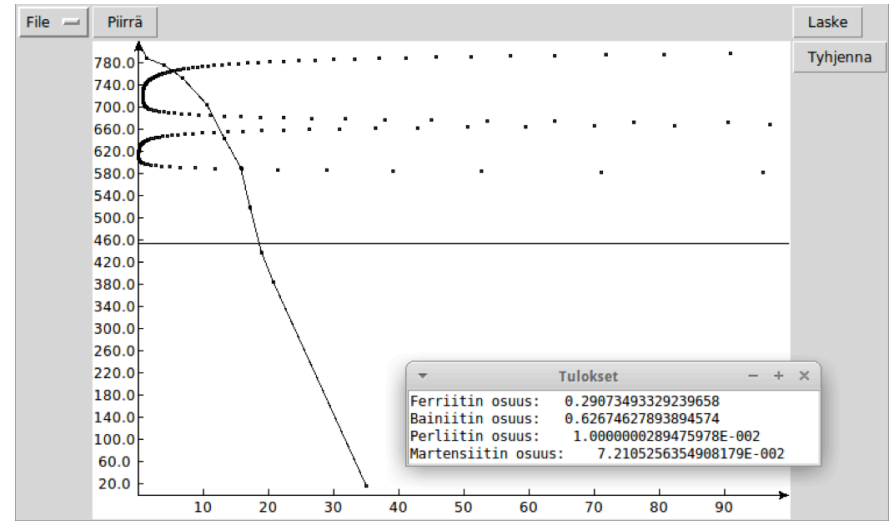

Figure 3. GUI for calculating phase transformations along user-defined cooling path. The user selects the path by clicking on the window and the phases formed can be calculated at any point along the path.

The GUI is shown in Figure 4. The temperature in the middle of the strip is shown in the graph window. The sliders are used to change the desired temperature. User can also load data from the more detailed model in order to compare this quick and approximate model to a more detailed data, calculated by the method described in section 3.1. The user can change the speed and thickness of the strip. Once the waters have been determined, the detailed model can be used to confirm the results and for fine-tuning, if necessary.

In addition to its main goal of determining the cooling water required to cool the strip to desired temperature, the approximate model can also be used in the same way as the detailed model described in section 3.1 to obtain quick and approximate estimate in few seconds (compared to minutes needed by the detailed model).

The GUI as well as the underlying numerical code was programmed with MATLAB. While the interaction of the code with the GUI is ideal within a single language, the non-compiled code is limited in speed. Although this was not a problem in the case described here, this limitation could be overcome by compiling some part of the code and interacting with it through the MATLAB software. (Matlab documentation)

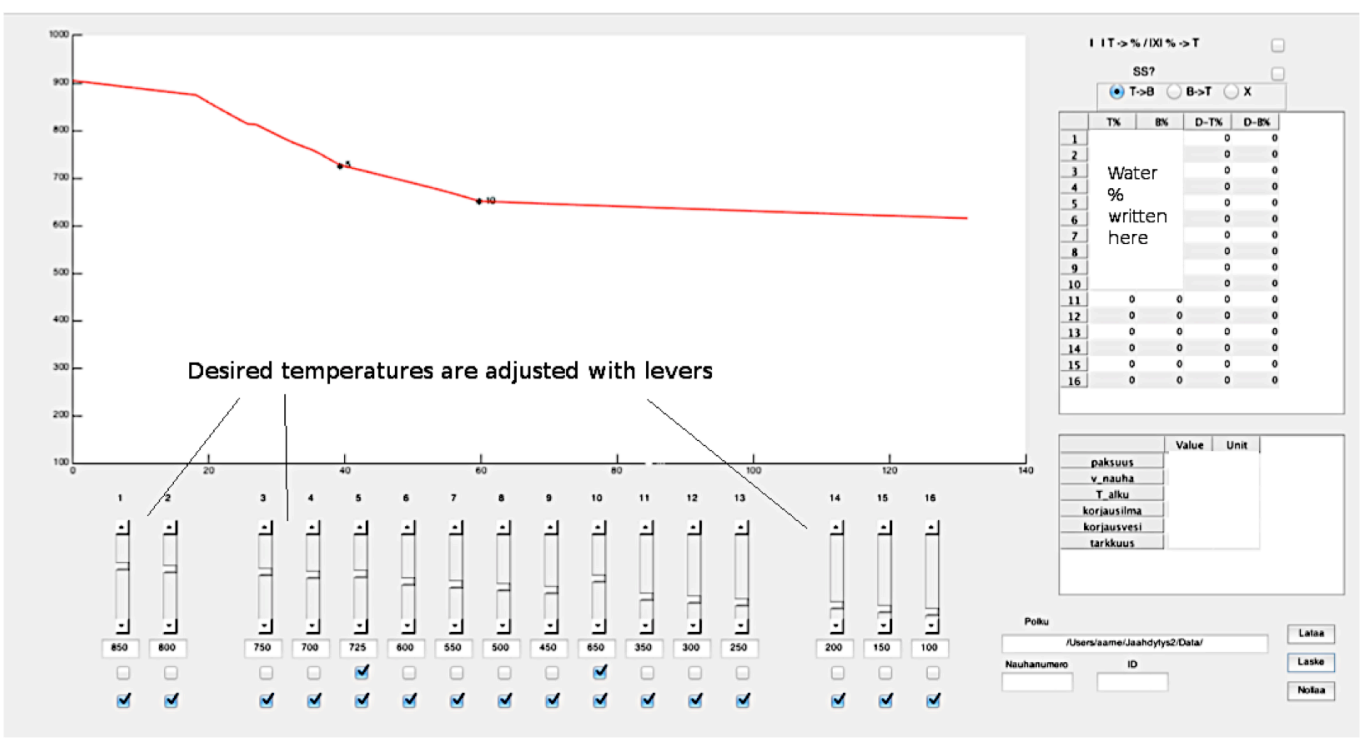

Figure 4. GUI for calculation of required cooling water usage to cool steel strip to desired temperature. The user adjusts the desired cooling water with the levers, defines strip thickness and speed. The required cooling water usage is written on the side panel. 


\section{Combined use of different tools and analytical approximations}

The tools presented in this article provide a way to design a suitable cooling water usage for a steel in order to achieve desired amount of final microstructural constituents (ferrite, bainite, pearlite, martensite). With the use of different tools that have different accuracy, the user can find a quick approximation, which can then be confirmed and finetuned with more detailed simulation.

While the heat conduction tool described in section 3.1 can be also fully coupled with the phase transformation tool described in section 3.2, the separate calculation of phase transformations as function of user-defined cooling path is also useful from the design point of view.

The use of different simulation tools and analytical approximation is further illustrated by the following cases

\subsection{Planning a cooling water usage to obtain desired amount of ferrite and bainite in the mid-depth}

In order to find a suitable cooling path that would produce desired amount of ferrite and bainite in the mid-depth of the strip/plate, we start with trials using the tool described in section 3.2. Assume that we wish to produce $40 \%$ ferrite, $40 \%$ bainite and $20 \%$ martensite for a given steel. Experimenting with different cooling rates, and calculating intermediate results, we can quickly find a suitable cooling rate shown in Figure 5.

After finding the desired cooling path, we wish to estimate the waters required to cool the mid-depth of the strip to this temperature. For this purpose we apply the tool described in section 3.3.

After calculating an estimate for the water usage we can confirm by using the tool described in section 3.1, that the more detailed model gives the same result as the quick estimate.

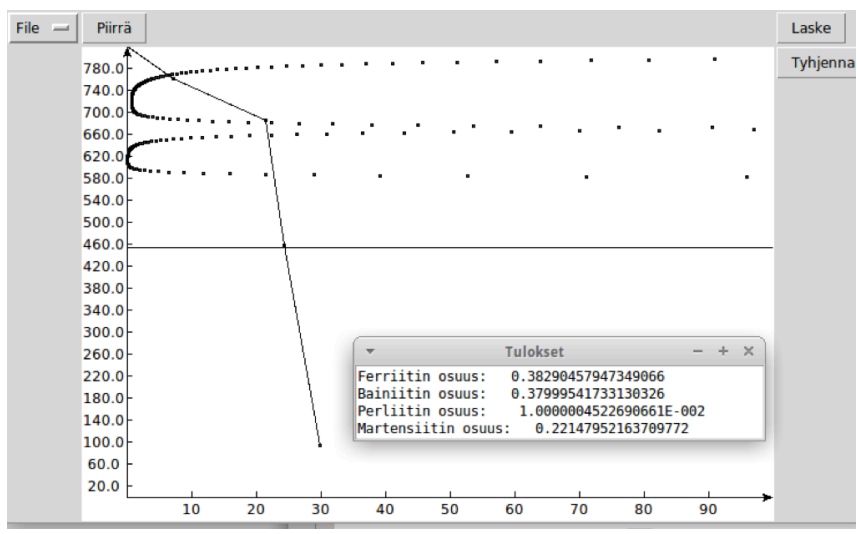

Figure 5. The desired cooling path to achieve roughly $40 \%$ ferrite, $40 \%$ bainite, and $20 \%$ martensite, calculated with the tool described in section 3.2 .

\subsection{Estimation of temperature distribution for given cooling rate and strip/plate thickness}

Since the temperatures in the surfaces can differ significantly from the temperature in the middle of the strip/plate, especially for thicker products, it is useful to understand how the strip thickness and cooling rate affect the temperature distribution within the strip and also to the time needed for the temperature distribution to relax towards the time-asymptotic temperature distribution as described in section 2. It is also useful for the user to be able to calculate quick estimates for the temperature distributions.

The equations presented in section 2 could be easily implemented to a computer algebra system such as the free and open source Maxima CAS (Computer Algebra System) (Maxima webpage) with a graphical user interface wxMaxima (wxMaxima webpage), Maple, Mathematica, or even modern handheld CAS enabled calculators. Sample wxMaxima syntax for the equations presented in this article is available from the first author of this article upon request, and will be made available through the author home page, www.iki.fi/aarne.pohjonen.

We use the following constant values for the thermal conductivity $\kappa=30 \mathrm{~W} /(\mathrm{m} \mathrm{K})$, density $\rho=$ $7400 \mathrm{~kg} / \mathrm{m}^{3}$, and specific heat capacity $c=700 \mathrm{~J} /(\mathrm{kg}$ $\mathrm{K})$ for the sample calculations. The constant $k$ in the equation (7) is then

$k=\kappa /(\rho c) \approx 5.8 \times 10^{-6}$.

We apply the equation (7) to calculate the timeasymptotic solution for the temperature distribution. For a $10 \mathrm{~mm}$ thick strip, $L=0.005$, subjected to constant cooling rates $c_{l}=-40^{\circ} \mathrm{C} / \mathrm{s}$ on the top and bottom of the strip with the cooling start temperature $c_{0}=1000^{\circ} \mathrm{C}$ the temperature distribution converges in $3 \mathrm{~s}$ towards the temperature distribution

$T_{A}(x, 3 \mathrm{~s})=966.3^{\circ} \mathrm{C}-3.45333 \times 10^{6} x^{2}$, where the depth coordinate $x$ is in the interval $-L, \ldots, L$ and the middepth is at $x=0$.

The time required to converge towards the solution can be estimated using the equation (11). Since $n=1$ gives the slowest convergence, the time constant can be calculated as $\tau_{l}=k \pi^{2} /\left(4 L^{2}\right) \approx 1.7 \mathrm{~s}$. This means that in order for the difference between the asymptotic and the current temperature distribution to diminish everywhere to at most $20 \%$ of the original difference, a time $t=-\ln (0.2) \times \tau_{1} \approx 2.8 \mathrm{~s}$ is required. 
Similar calculations can be applied together with the procedure illustrated with case 4.1 in order to estimate the temperature distributions inside of the strip, when the strip is cooled along the different cooling paths.

Equation (7) shows that the difference between the surface temperature and the mid-depth temperature in the time-asymptotic solution is $c_{l} L^{2} /(2 k)$, i.e. linear dependence on cooling rate and propotional to $L^{2}$. The equations (9) (10) (11) show that the time required for the temperature distribution to converge to the timeasymptotic solution is proportional to $L^{2}$. Although the simulation tool 3.1 can be used to calculate the timedependent temperature distribution for the strip of any thickness and cooling rate, the analytical approximation gives immediately the idea how changing the thickness and cooling rate affect the results.

\subsection{Effect of different cooling rates on the top and bottom to the temperature distribution}

The flatness of a steel plate is an important property, which should be optimized for the final product. If the temperature distribution of the steel plate is such that other side of the plate is at higher temperature during cooling, the difference in phase transformation start times and thermal expansion can lead to very poor flatness quality.

To see how the different cooling rates on top and bottom of the plate affect the temperature distribution inside of the plate, we apply equation (6) to calculate the time asymptotic temperature distribution when the other side of the plate is cooled with rate $c_{I}=-15^{\circ} \mathrm{C} / \mathrm{s}$ and the other with rate $d_{l}=-10^{\circ} \mathrm{C} / \mathrm{s}$. We assume 30 $\mathrm{mm}$ thick plate, $L=0.015$. The time asymptotic temperature distribution after $20 \mathrm{~s}$ of cooling starting with $1000^{\circ} \mathrm{C}$ initial temperature, calculated with (6) is shown in Figure 6.

\section{Conclusions}

We present analytical approximations and simulation tools that can be used for designing cooling path and water usage to cool hot rolled steel strip in order to achieve desired amounts of ferrite, pearlite, bainite and martensite, and understanding how different factors affect the results. The usage of the analytical approximations and the simulation tools is shown by different example cases described in section 4.

\section{Acknowledgements}

Useful discussions on the actual dimensions of the steel strips/plates with M.Sc (tech) Olli Leinonen are acknowledged.

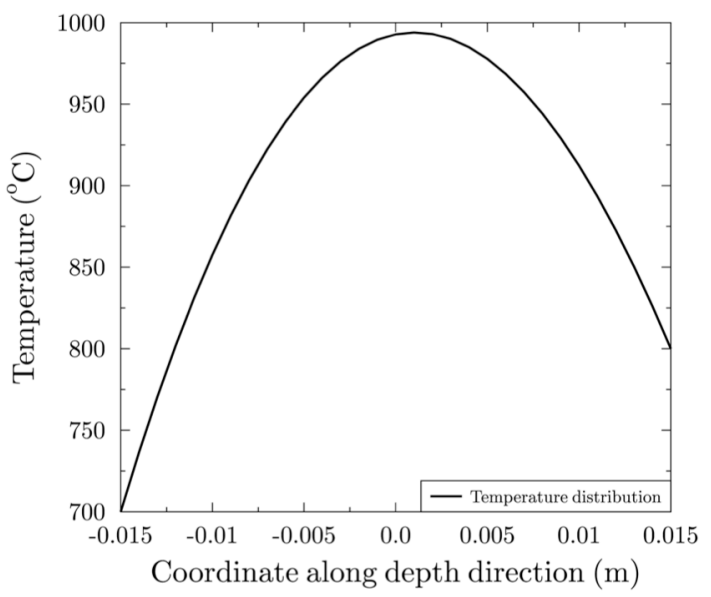

Figure 6. Uneven time-asymptotic temperature distribution $T_{C}(x, 20 \mathrm{~s})$ caused by difference between the cooling rates on the top and bottom of the strip/plate.

\section{References}

H.S. Carslaw and J.C. Jaeger. Conduction of Heat in Solids, $2^{\text {nd }}$ edition, Clarendon Press, Oxford, 1989, p.102-105.

Matlab documentation, http://se.mathworks.com/help/matlab/.

Maxima CAS open source computer algebra system, http://maxima.sourceforge.net.

J. Paananen. Laskennallinen työkalu kuumavalssatun teräsnauhan jäähdyttämisen suunnitteluun, Oulun yliopisto, Bachelors Thesis, 2015.

A. Pohjonen, M. Somani, J. Pyykkönen, J. Paananen, and D. Porter. The Onset of the Austenite to Bainite Phase Transformation for Different Cooling Paths and Steel Compositions, Key Engineering Materials, 716: 368-375, 2016.

Python documentation, https://www.python.org/doc/.

J. M. Pyykkönen, D. C. Martin, M. C. Somani and P. T. Mäntylä. Thermal behaviour of steel plate during accelerated cooling, Materials Science Forum, 638-642: 2706-2711, 2010.

J. Pyykkönen, M. Somani, D. Porter, M. Holappa and T. Tarkka. Modelling of Thermal History and Microstructural Evolution on the Run-out Table of a Hot Strip Mill, Proceedings from the $6^{\text {th }}$ International Quenching and Distortion Conference: 817-828, $2012 \mathrm{a}$.

J. Pyykkönen, M. Somani and D. Porter. Experimental and Simulation Studies of Thermal and Microstructure Evolution During Accelerated Cooling of Advanced High Strength Steels, ROLLING 2013, 9th International ROLLING Conference \& 6th European ROLLING Conference: 1-11, 2013.

J. Pyykkönen, P. Suikkanen, M. Somani and D. Porter. Effect of temperature, strain and interpass time on microstructural evolution during plate rolling, Journées Annuelles de la SF2M 2012 / SF2M Annual Meeting 2012, Colloque 1, S1: 17-19, 2012 b.

wxMaxima, open source graphical frontend to Maxima CAS, http://andrejv.github.io/wxmaxima/. 